\title{
ACTIVE-ONLY SINUSOIDAL OSCILLATOR WITH ELECTRONICALLY-TUNABLE FULLY-UNCOUPLED FREQUENCY AND CONDITION OF OSCILLATION
}

\author{
MUHAMMAD TAHER ABUELMAATTI \\ King Fahd University of Petroleum and Minerals, Box 203, Dhahran 31261, \\ Saudi Arabia
}

(Received 8 September 2001; In final form 6 November 2001)

\begin{abstract}
A new active-only sinusoidal oscillator is presented. The oscillator circuit uses two internally compensated operational amplifiers, two plus-type second-generation current conveyors and three operational transconductance amplifiers. The proposed circuit enjoys the attractive features of totally uncoupled frequency and condition of oscillation, low sensitivities, electronic tunability and integratability.
\end{abstract}

\section{INTRODUCTION}

At present, there is a growing interest in designing capacitorlessresistorless active-filters and oscillators using only active-elements, such as operational amplifiers (OAs), operational transconductance amplifiers (OTAs) and current-conveyors (Tsukutani et al., 1996; Abuelmaatti and Al-Zaher, 1997;1998a;1998b; Tsukutani et al., 2000). This is attributed to their integratability, programmability and wide frequency range of operation.

Active-only resistorless-capacitorless sinusoidal oscillators using two OAs only have been reported in the literature (Abuelmaatti and Almansoury, 1986; Bhat and Shah, 1989). While there is no control on the condition of oscillation, and consequently the amplitude of oscillation, these oscillator circuits suffer from the additional disadvantage 
of the need to change the d.c. supply of the whole circuit in order to change the frequency of oscillation.

An active-only sinusoidal oscillator circuit is reported in (Abuelmaatti and Al-Zaher, 1998). This circuit was obtained by converting an RLC resonator into an RLM resonator by scaling all the impedances of the RLC resonator by $s / \omega$ where $\mathrm{M}$ is a FDNC characterized by $Z(s)=M s^{2}$. In this paper, it will be shown that active only sinusoidal oscillators can be directly obtained from an RLC resonator comprising an inductor, a capacitor and two resistors. First, new active-only simulators will be proposed for grounded inductors and capacitors. Then, these new simulators will be combined with activeonly simulated grounded positive and negative resistances to obtain a sinusoidal oscillator circuit.

\section{PROPOSED CIRCUITS}

Consider the circuit shown in Figure 1(a). Using standard notations, the OTA can be characterized by $i=g_{m k}(v-v)$ where $g_{m k}=I_{A B C k} / 2 V_{T}$ is the transconductance of the $k$ th OTA, $I_{A B C k}$ is its auxiliary biascurrent, $V_{T}$ is the thermal voltage and $v$ and $v$ are the input voltages of the OTA. The plus-type second-generation current-controlled currentconveyor (CCCCII) can be characterized by $i_{y}=0, V_{x}=V_{y} R_{x} i_{x}$ and $i_{z}=i_{x}$ where $R_{x}=V_{T} / 2 I_{o}$ and $I_{o}$ is the bias current of the CCCCII. Assuming internaly compensated OAs with open-loop gain of $A_{k}=B_{k} / s$, where $B_{k}$ is the gain-bandwidth product of the $k$ th OA, routine analysis yields the input impedance given by

$$
Z_{\text {in } 1}=\frac{s R_{x 1}}{B_{1}}
$$

Thus, the circuit of Figure 1(a) realizes a lossless grounded inductor $L_{\text {eq }}=R_{x \mathrm{l}} / B_{1}$. It is worth mentioning here that the circuit of Figure 1(a) can be obtained from the circuit of Figure 3 of Khan et al., 1992, simply by replacing the OTA by the CCCCII. The advantages of using the CCCCII instead of the OTA are as follows:

a. For the conventional bipolar OTA the value of $g_{m}$ is $I_{\text {Bias }} / 2 V_{T}$, while $1 / R_{x}$ for the CCCCII is $2 I_{\text {Bias }} / V_{T}$. Thus for the same 

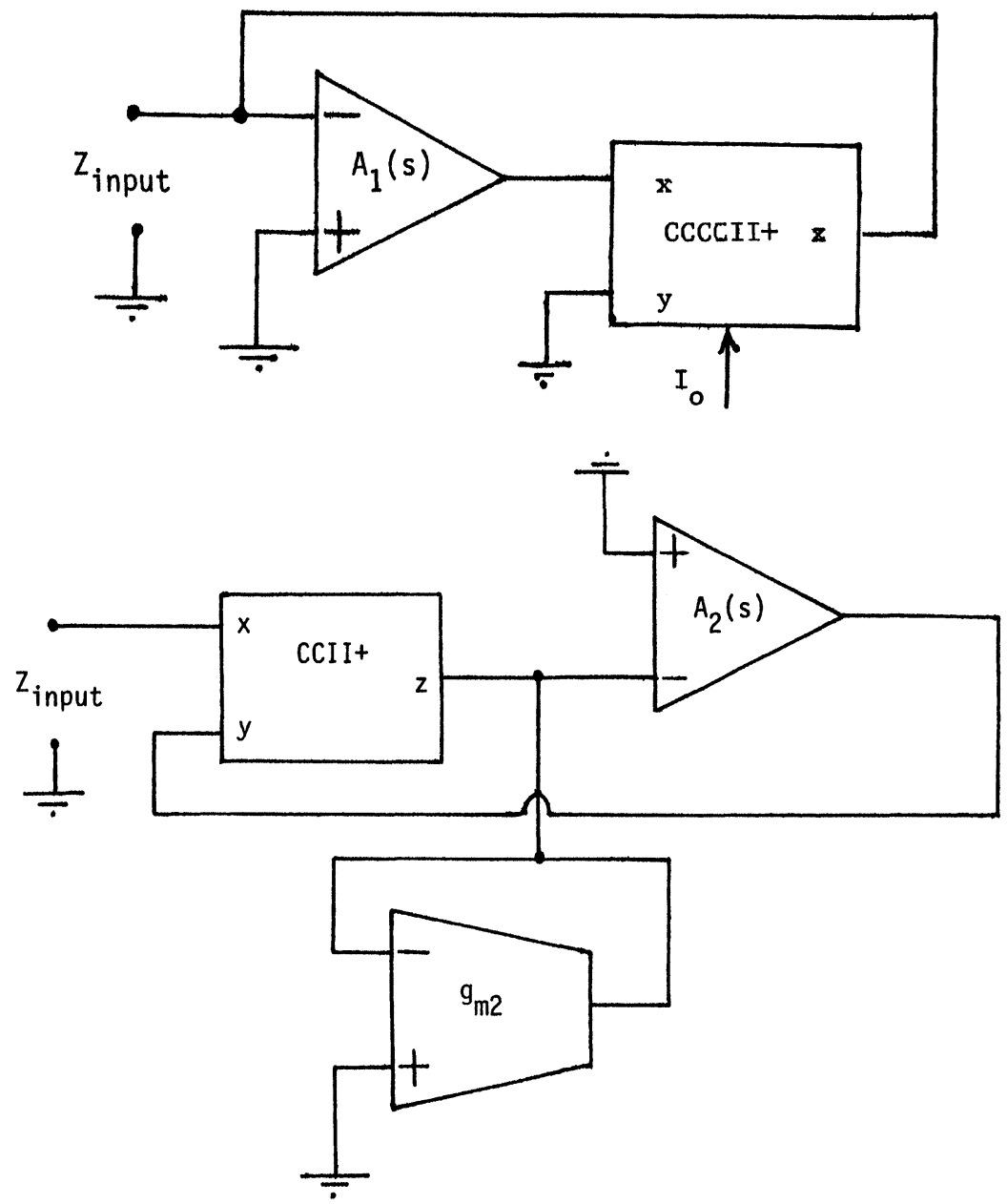

FIGURE 1 Proposed simulators for (a) grounded inductor and (b) grounded capacitor.

biasing current $I_{\text {Bias }}$, the transconductance of the OTA will be 4 times less than that of the CCCCII. This means that an OTA implementation may result in a higher power consumption.

b. Because of the use of high values of currents, the maximum frequency usable for an OTA-based implementation will be reached sooner than that for a CCCCII based implementation. 
The plus-type second-generation current-conveyor (CCII) can be characterized by $i_{y}=0, V_{x}=V_{y}$ and $i_{z}=i_{x}$. Routine analysis shows that the input impedance of the circuit of Figure 1(b) can be expressed as

$$
Z_{\text {in } 1}=\frac{1}{s g_{m 2} / B_{2}}
$$

Thus, the circuit of Figure 1(b) realizes lossless grounded capacitor $C_{\mathrm{eq}}=g_{m 2} B_{2}$. It is worth mentioning here that the OTA in the circuit of Figure 1(b) can be replaced by a CCCCII with its y-and z-terminals connected to ground, and its $\mathrm{x}$-terminal connected to the $\mathrm{z}$-terminal of the CCII. It is interesting to note also that if the parasitic input resistance of the CCII of Figure 1(b) is taken into consideration, then the input impedance will be formed of a series combination of the capacitor of Eq. (2) and a resistor $R_{x}$. Minimizing this resistance is possible using the composite second-generation current-conveyor with reduced parasitic resistance (Fabre and Barthelemy, 1994).

Combining the circuits of Figure 1 yields a sinusoidal oscillator circuit with frequency of oscillation given by

$$
\omega_{o}^{2}=\frac{1}{L_{\mathrm{eq}} C_{\mathrm{eq}}}=B_{1} B_{2} \frac{1}{g_{m 2} R_{x 1}}
$$

This oscillator circuit suffers from the disadvantage of having no condition of oscillation and consequently no control on the amplitude of oscillation. This can be avoided by introducing two OTAs configured as grounded positive and negative resistances as shown in Figure 2. Thus the condition of oscillation of the circuit of Figure 2 will be given by

$$
g_{m 3}=g_{m 4}
$$

From Eqs. (3) and (4) it appears that the frequency of oscillation and the condition of oscillation are totally uncoupled, that is while the frequency of oscillation is controlled by a set of parameters, the condition of oscillation is controlled by a completely different set of parameters. This property is very attractive for electronic tuning of the frequency and the amplitude of oscillation. Moreover, it appears that while $R_{x 1}, g_{m 2}, g_{m 3}$ and $g_{m 4}$ are temperature dependent, the frequency 


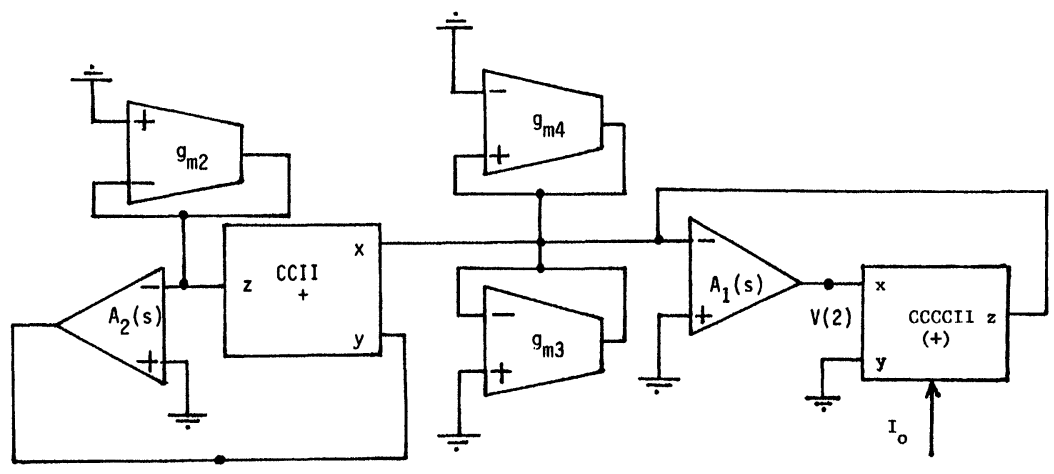

$V(4)$

FIGURE 2 Proposed active-only totally-uncoupled electronically-tunable sinusoidal oscillator circuit.

and the condition of oscillation are temperature insensitive. Finally, it is interesting to note that the circuit can support two low-output impedance quadrature voltages.

From Eq. (3) it is easy to show that the sensitivities of the parameter $\omega_{o}$ are:

$$
\begin{aligned}
& S_{B_{1}}^{\omega_{o}}=S_{B_{2}}^{\omega_{o}}=-S_{R_{x 1}}^{\omega_{o}}=-S_{g_{m 2}}^{\omega_{o}}=\frac{1}{2} \\
& S_{g_{m 3}}^{\omega_{o}}=S_{g_{m 4}}^{\omega_{o}}=0
\end{aligned}
$$

all of which are small.

\section{SIMULATION RESULTS}

The sinusoidal oscillator circuit of Figure 2 was simulated using the PSPICE circuit simulation program. The OA 741 with corner frequencies $f_{1}=10 \mathrm{~Hz}, f_{2}=3 \mathrm{MHz}$, input resistance $R_{\text {input }}=400 \mathrm{k}$ output resistance $R_{\text {output }}=200$ and DC gain $=100000$, the OTA macromodel [Wu, 1994] and the current conveyor macromodel [Svoboda, 1994] were used in simulation. Figure 3 shows typical output waveforms obtained with $1 / R_{x 1}=g_{m 2}=g_{m 3}=1 \mathrm{~mA} / \mathrm{V}, g_{m 4}=1.1 \mathrm{~mA} / \mathrm{V}$ and the DC supply voltage $=5 \mathrm{~V}$. Figure 4 shows the output spectrum of the waveforms of Figure 3. From Figure 3 it is easy to see that the voltages 


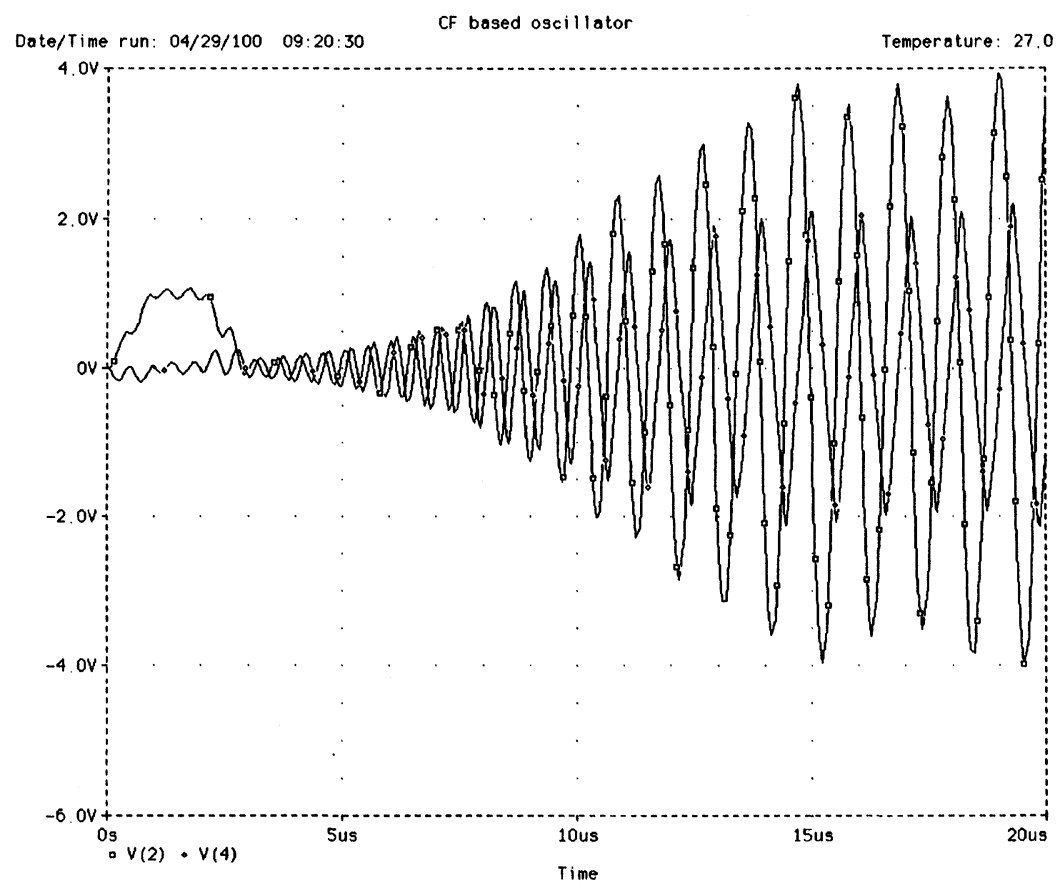

FIGURE 3 Simulated output waveforms obtained with $1 / R_{x 1}=g_{m 2}=g_{m 3}=1 \mathrm{~mA} / \mathrm{V}$, $\mathrm{g}_{m 4}=1.1 \mathrm{~mA} / \mathrm{V}$ and the DC supply voltage $=5 \mathrm{~V}$.

$\mathrm{V}(2)$ and $\mathrm{V}(4)$ are in quadrature. Figure 5 shows the variation of the frequency of oscillation with the parameter $1 / R_{x 1}$ while $g_{m 2}=$ $g_{m 3}=1 \mathrm{~mA} / \mathrm{V}, g_{m 4}=1.1 \mathrm{~mA} / \mathrm{V}$.

\section{CONCLUSION}

A new active-only sinusoidal oscillator with totally uncoupled frequency and condition of oscillation has been presented. The new oscillator circuit is obtained directly from the RLC resonator without recourse to the classical approach of obtaining an RLM circuit from the RLC resonator. Direct realization of the new oscillator circuit required the development of a new active-only grounded inductor and a new active-only grounded capacitor. Combining these new active 


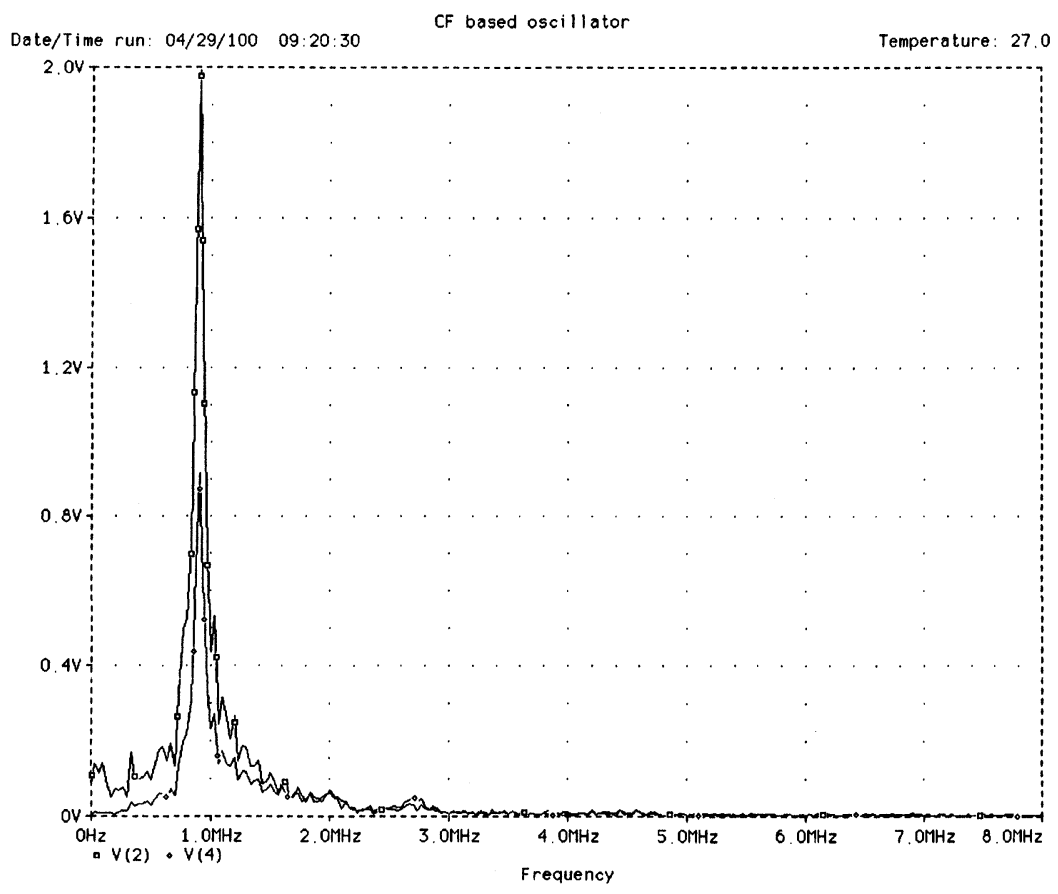

FIGURE 4 Spectrum of the output waveforms of Figure 3.

only inductor and capacitor with OTA-based grounded positive and negative resistances, the new oscillator circuit was obtained.

In addition to using only active devices for its realization, the new circuit enjoys the following advantages:

1. Totally uncoupled frequency and condition of oscillation.

2. Low sensitivities of the frequency of oscillation to the gainbandwidth products of the OAs, the transconductances of the OTAs and the parasitic resistance of the CCCCII.

3. Despite the temperature dependence of the transconductances of the OTAs and the parasitic resistance of the CCCCII, the frequency of oscillation and the condition of oscillation are temperature insensitive.

4. The circuit supports two low output-impedance quadrature voltages. 


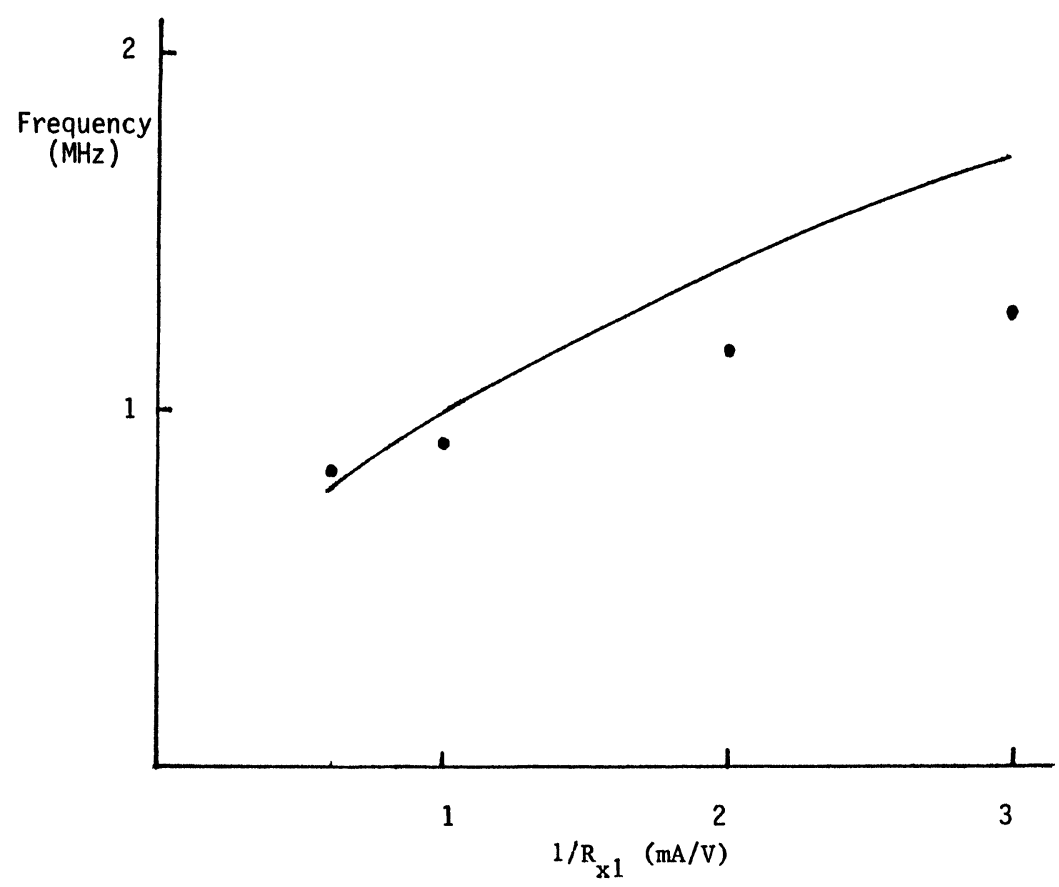

FIGURE 5 Variation of the frequency of oscillation with the parameter $1 / R_{x 1}$ while $g_{m 2}=g_{m 3}=1 \mathrm{~mA} / \mathrm{V}, g_{m 4} 1.1 \mathrm{~mA} / \mathrm{V}$.

$\stackrel{2}{-}$ Calculated Eq. (3) $\bullet$ : Simulated.

\section{References}

Abuelmaatti, M. T. and Al-Zaher, H. A. (1997). Universal three input and one output current mode filter without external passive elements. Electronics Letters, 33, 281283.

Abuelmaatti, M. T. and Al-Zaher, H. A. (1998a). Multi-function active-only currentmode filter with three inputs and one output. International Journal of Electronics, $85,431-435$.

Abuelmaatti, M. T. and Al-Zaher, H. A. (1998b). Active-only sinusoidal oscillator. Microelectronics Journal, 29, 461-464.

Abuelmaatti, M. T. and Almansoury, W. A. (1986). New active-R sinusoidal oscillator. International Journal of Electronics, 60, 771-775.

Bhat, C. K. and Shah, N. A. (1989). A novel voltage controlled oscillator. Electronic Engineering, 61, 25-26.

Fabre, A. and Barthelemy, H. (1994). Composite second-generation current conveyor with reduced parasitic resistance. Electronics Letters, 30, 377--378.

Khan, I. A., Ahmed, M. T. and Minhaj, N. (1992). Novel technique for immittance simulation-realisation of some all-active simulators. International Journal of Electronics, 72, 431-441. 
Svoboda, J. A. (1994). Comparison of RC op.-amp. and RC current conveyor filters. International Journal of Electronics, 76, 615-626.

Tsukutani, T., Ishida, M., Tsuiki, S. and Fukui, Y. (1996). Current-mode biquad without external passive elements. Electronics Letters, 32, 197-198.

Tsukutani, T., Higashimura, M., Sumi, Y. and Fukui, Y. (2000). Electronically tunable current-mode active-only biquadratic filter. International Journal of Electronics, 87, 307-314.

Wu, J. (1994). Current-mode high-order OTA-C filters. International Journal of Electronics, 76, 1115-1120. 

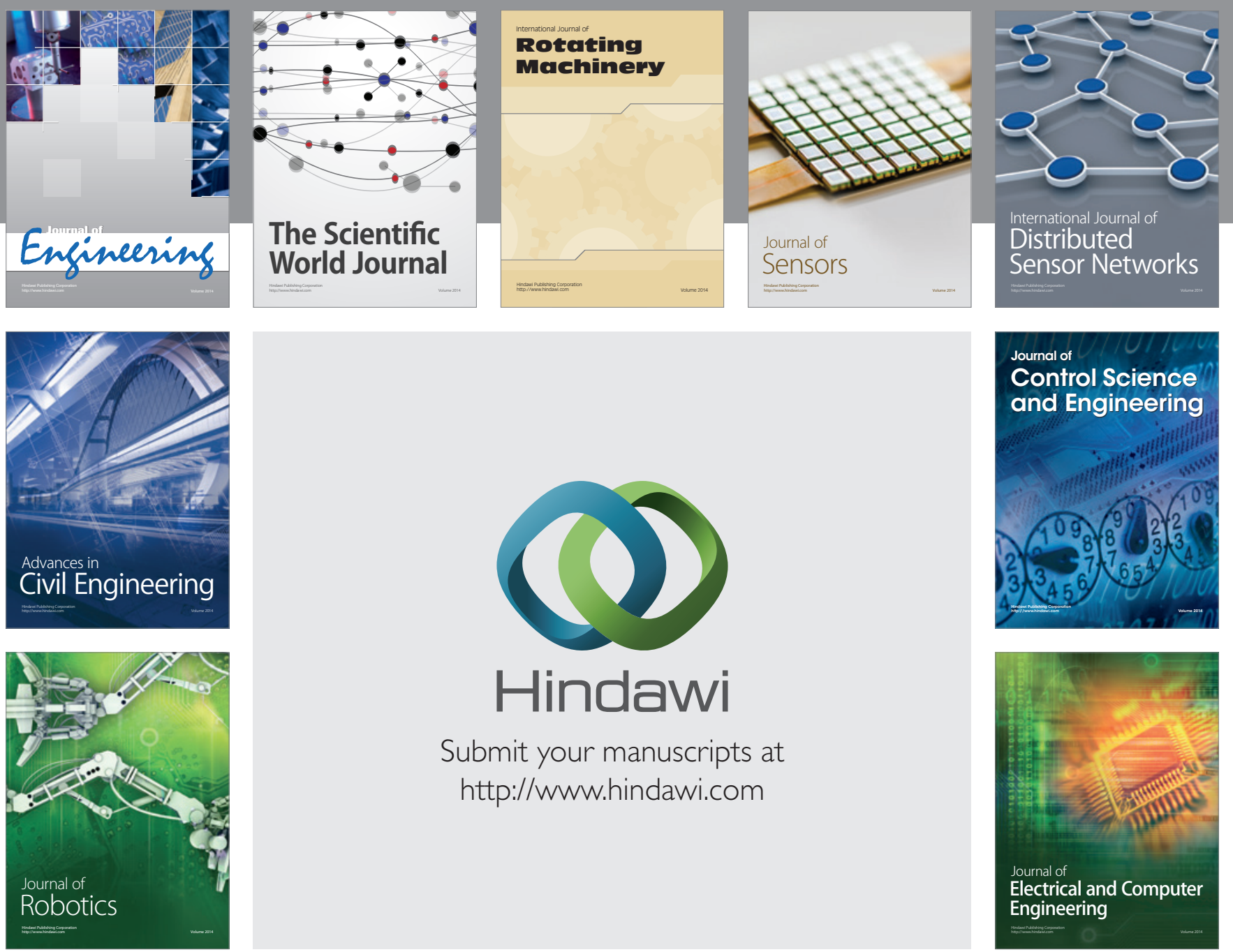

Submit your manuscripts at

http://www.hindawi.com
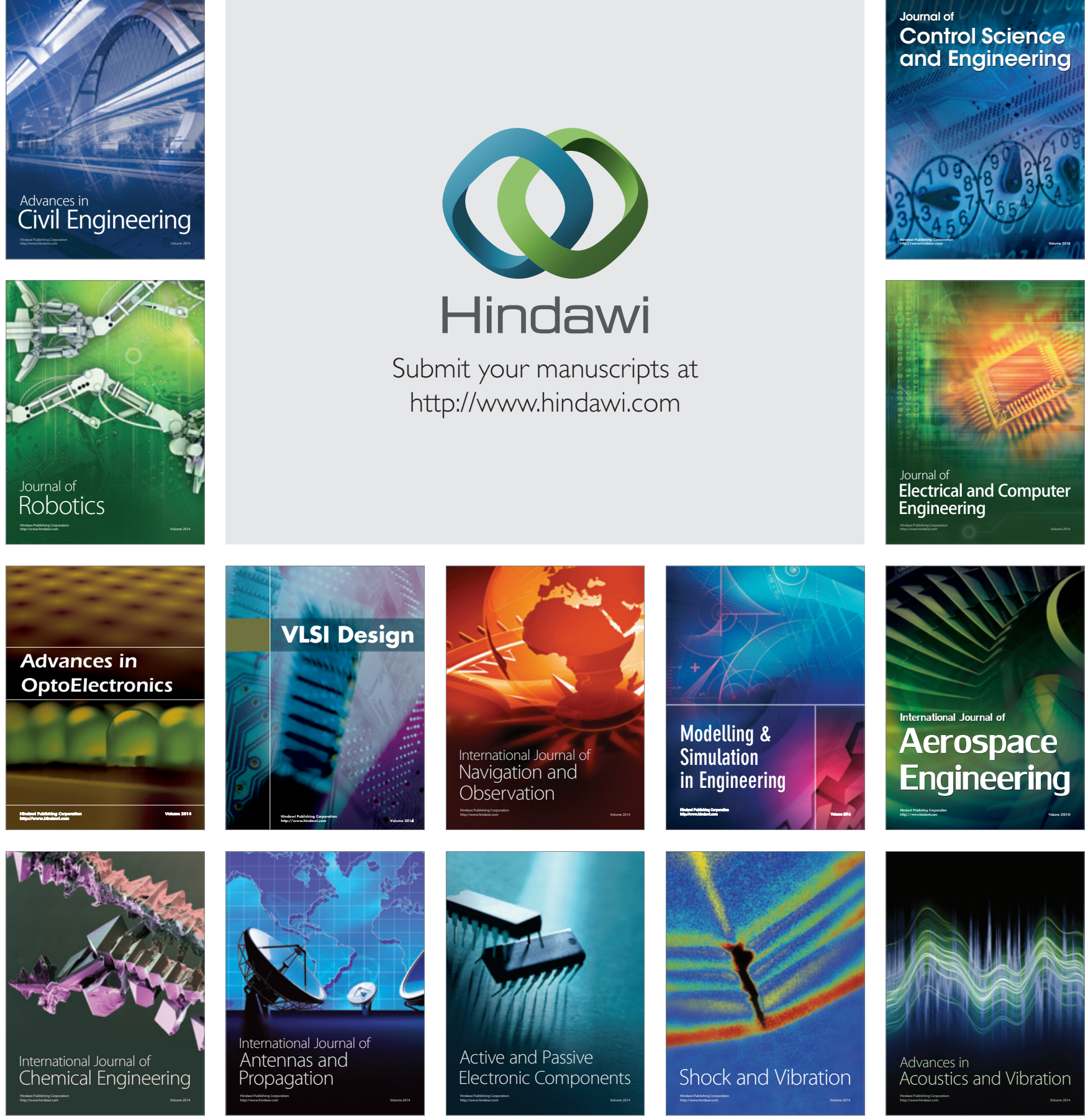